\title{
Residual plaque prolapse with novel dual-layer carotid stents: is it mesh-covered or not?
}

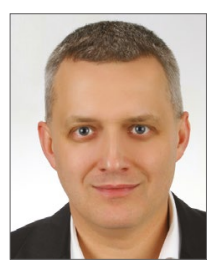

Piotr Musialek ${ }^{1}, \mathrm{MD}$, DPhil; Eugenio Stabile², MD, PhD

\begin{abstract}
1. Jagiellonian University Department of Cardiac \& Vascular Diseases, John Paul II Hospital, Krakow, Poland; 2. Division of Cardiology, Department of Advanced Biomedical Sciences, University of Naples "Federico II", Naples, Italy
\end{abstract}

With the long-term equipoise of carotid artery stenting (CAS) and carotid endarterectomy (CEA) demonstrated unequivocally in CREST and other large studies, the debate between CAS and CEA today is about the (mostly minor) strokes within the first 30 days after the procedure. With conventional (single-layer) carotid stents, the risk for cerebral embolisation (unlike in CEA) continues postprocedurally ${ }^{1}$, i.e., when the embolic protection device is no longer able to capture the debris. As $40-80 \%$ of strokes associated with conventional-stent CAS are post-procedural, minimisation of periprocedural embolism and elimination of post-procedural embolism are critical for the future of $\mathrm{CAS}^{2}$.

With conventional carotid stenting, plaque prolapse $\mathrm{e}^{3-5}$ - a major mechanism of post-procedural stroke ${ }^{5}$ - occurs via the "cheese-grater" effect, in a phenomenon similar to that observed with single-layer coronary stents ${ }^{6}$. To address the problem of plaque prolapse in CAS, mesh-covered carotid stents have been developed and introduced into routine clinical practice ${ }^{2,7}$. The "ultra-closed cell" carotid stent design is produced by covering the nitinol frame with a mesh that can be made of different materials $^{7-9}$. At present, the two mesh-covered stents in clinical use are RoadSaver ${ }^{\circledR}$ (aka Casper; MicroVention/Terumo [Tokyo, Japan $])^{8}$ and CGuard ${ }^{\text {TM }}$ EPS (InspireMD, Boston, MA, USA) $)^{9,10}$. Apart from the differences in the nitinol frame (braided closed cell in RoadSaver/Casper ${ }^{7,8}$, laser-cut open cell in $\mathrm{CGuard}^{7,9}$ ), the devices have three important differences in relation to the mesh coverage: (i) the mesh material is different (braided nitinol in RoadSaver/Casper, PET Micronet in CGuard EPS); (ii) the mesh pore aperture size is different (375-500 $\mu \mathrm{m}$ in RoadSaver/ Casper vs. 150-180 $\mu \mathrm{m}$ in CGuard EPS); and (iii) the position of the mesh in relation to the nitinol frame is different (outside the frame for the CGuard EPS, and inside in the case of the RoadSaver/Casper ${ }^{7-9}$. Indeed, with the MicroNet covered carotid stent systematic per protocol DW-MRI evaluation demonstrated minimisation of intraprocedural embolisation and elimination of post-procedural cerebral embolisation ${ }^{10}$. This strategy has been termed intraprocedural and post-procedural (sustained) "embolic prevention" in $\mathrm{CAS}^{10,11}$.

In this issue of EuroIntervention, Umemoto and colleagues ${ }^{12}$ report outcomes of CAS patients treated with two different meshcovered stents.

\section{Article, see page 1348}

Endpoints of interest included the occurrence of stent malapposition (defined as at least five malapposed struts in a single OCT slice and with the strut defined as "malapposed" when the distance between vessel wall and the strut surface exceeded $200 \mu \mathrm{m}$ ) and plaque prolapse (defined as tissue protrusion more than $300 \mu \mathrm{m}$ from the stent strut level). On slice-based analysis, stent malapposition occurred in $26.8 \%$ with RoadSaver vs. $20.5 \%$ with CGuard EPS $(p=0.26)$ and plaque prolapse in $20.7 \%$ and $10.8 \%$, respectively $(\mathrm{p}=0.05)$.

The messages from the work by Umemoto and colleagues ${ }^{12}$, indicating a similar rate of stent malapposition with the two designs and an approximately twofold greater risk of residual plaque prolapse with the dual metallic layer device, deserve 
further investigation. This is not only because, ideally, one would want to see a larger study in patients with well-defined baseline plaque characteristics ${ }^{6}$, but also because this work illustrates some fundamental limitations of the current definitions and techniques when used to evaluate the novel devices. For stent malapposition, in an appropriately sized open-cell stent ${ }^{9,11}$, malapposition may be minimised by optimising post-dilatation. This, however, is more difficult to achieve with the dual-layer braided closed-cell design of the nitinol stent frame ${ }^{8}$. Stent malapposition will depend on stent expansion optimisation in the context of stent design (open vs. closed-cell frame) and the stent nominal diameter in relation to the vessel diameter. Initial data indicate that open-cell mesh-covered stents may allow more robust optimisation ${ }^{11}$.

It is important to realise that the differences in design of the two devices $^{8,9}$, including the mesh material and mesh position in relation to the stent frame, translate into completely different types of plaque prolapse. In RoadSaver/Casper, the braided metallic mesh of $45 \mu \mathrm{m}$-diameter of nitinol filament is fixed (at many fixation points ${ }^{8}$ ) inside the braided stent frame and, because its diameter significantly exceeds the axial resolution of optical coherence tomography (OCT), the mesh is routinely visualised on OCT (see Figure $\mathbf{6 A}$ in ref. 12 and the schematic presentation in Figure 1 below), and the OCT-detected plaque prolapse ${ }^{12}$ represents atherosclerotic plaque that is not mesh-covered (schematic presentation in Figure 1A below, for raw OCT images see Figure 6B in ref. 12). In contrast, in the CGuard EPS, the mesh, that is fixed to the stent frame only at its edges, can adapt flexibly to the plaque prolapse through the highly open cells of the CGuard EPS stent frame, with mesh coverage of the plaque protruding into the vessel lumen (see Figure 1A below, compare with a raw OCT image in Figure 7 in ref. 12). As found by Umemoto and colleagues ${ }^{12}$, conventional OCT may, in some slices, indicate mesh coverage of the prolapsing atherosclerotic plaque in CGuard EPS (cf. Figure 7 in ref. 12). Indeed, mesh coverage of protruding plaque prevents embolism

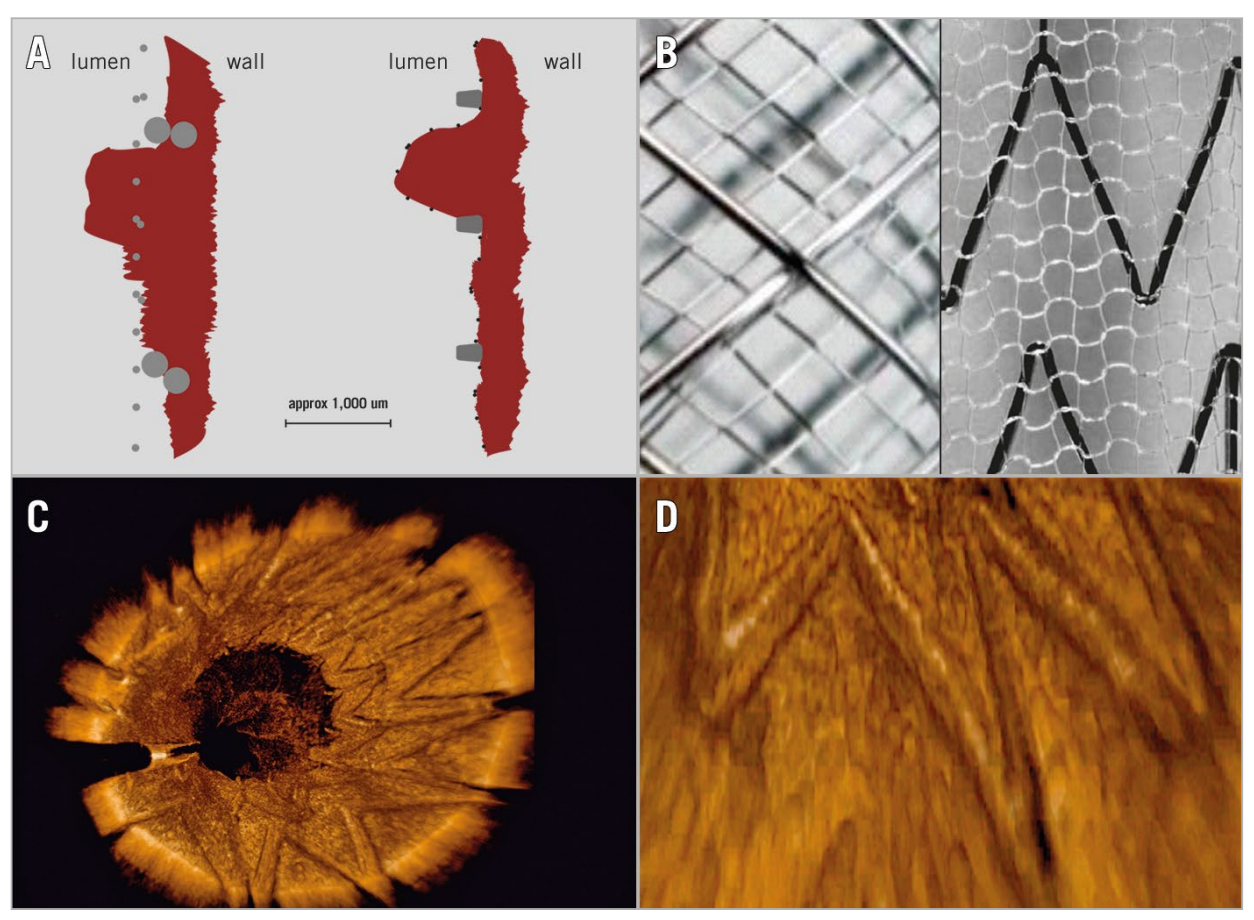

Figure 1. Residual plaque prolapse through mesh-covered carotid stent types in the context of fundamental stent design differences. A) Schematic presentation of plaque prolapse (PP). Left: schematic representation of a carotid stent with metallic mesh made of braided nitinol filaments $47 \mu \mathrm{m}$ in diameter (filament-to-filament distance of ca. $375 \mu \mathrm{m}$ ) fixed (with fixation points along the stent length) ${ }^{8}$ inside the stent frame that is made of braided nitinol wires of $180 \mu \mathrm{m}$. Right: schematic representation of a carotid stent with PET-fibre MicroNet, positioned outside the stent frame and fixed to the frame only at the proximal and distal stent edge that has the ability to adapt to the plaque, covering any protrusion (cf. Figure 7 in ref. 12) that may occur between the widely open laser-cut nitinol struts of $92 \times 125 \mu$ m. The PET-fibre diameter is only $20 \mu \mathrm{m}$ - beyond the effective resolution of conventional OCT. Nitinol is shown in grey, PET in black, and the plaque in dark red. B) Photographs (abluminal view) of the two dual-layer carotid stent designs available for commercial use today. Left: RoadSaver/Casper (braided nitinol closed-cell frame and braided nitinol closed-cell mesh that is fixed inside the stent frame). Right: CGuard EPS (laser-cut open-cell nitinol frame with knitted PET MicroNet outside the stent frame). According to refs 7 and 9, modified. C) 3D OCT reconstruction of the CGuard EPS implanted into a symptomatic human carotid artery. Note a clear lumen definition in the absence of PP (minor opacifications in the lumen are artefacts from residual contrast agent). According to ref. 7, modified. D) Detailed section from panel C. Note that the MicroNet PET fibres (20 $\mathrm{mm}$ in diameter) are beyond the effective resolution of conventional OCT; thus, the mesh is only barely indicated behind the stent frame (i.e., between the stent frame and the vessel wall). According to ref. 7, modified. 
by "trapping" the plaque in situ. However, we cannot be absolutely sure that prolapsing plaque is mesh covered - the CGuard EPS mesh is made of PET fibre that is only $20 \mu \mathrm{m}$ in diameter ${ }^{9}$, which is too small to permit systematic visualisation on conventional OCT (Figure 1C, Figure 1D).

Unfortunately, the findings of Umemoto and colleagues ${ }^{12}$ cannot be directly compared with the recent paper of Nerla et al ${ }^{13}$, because of the per-slice analysis in one study and the per-patient analysis in the other. Ideally, both per-patient and per-slice data should be provided because each conveys different information, and for future studies it would be valuable if this approach could be standardised. Also, the extent of the plaque prolapse (in terms of its area and "depth") may confer different risks of cerebral embolism. Further investigation of this issue is a matter of considerable interest.

Future efforts will need not only to evaluate plaque prolapse predictors $^{6}$ in the novel mesh-covered carotid stents but also to determine whether there is any definable unprotected (i.e., not covered/excluded by micromesh) plaque prolapse threshold for subclinical vs. clinical cerebral embolism. Moreover, rapidly evolving innovations will improve intravascular imaging resolution, enabling routine MicroNet filament visualisation. The research cycle of mesh-covered carotid stents (that now expands to large-scale clinical studies with a long-term follow-up ${ }^{2}$ ) may need re-evaluation in animal models, with progress in imaging technologies ${ }^{14,15}$ in a position to demonstrate unequivocally that the residual plaque prolapse seen with CGuard EPS is indeed mesh covered.

\section{Conflict of interest statement}

P. Musialek was co-Principal Investigator in the InspireMD CARENET Trial (Carotid Embolic Protection Using MicroNet) and participated in the CGuard Advisory Board. Currently he is a Principal Investigator in the industry-independent PARADIGM/ PARADIGM-Extend Academic Study. E. Stabile has no conflicts of interest to declare.

\section{References}

1. Schofer J, Arendt M, Tübler T, Sandstede J, Schlüter M. Late cerebral embolization after emboli-protected carotid artery stenting assessed by sequential diffusion-weighted magnetic resonance imaging. JACC Cardiovasc Interv. 2008;1:571-7.

2. Musialek P, Hopkins LN, Siddiqui AH. One swallow does not a summer make but many swallows do: accumulating clinical evidence for nearly-eliminated peri-procedural and 30-day complications with mesh-covered stents transforms the carotid revascularisation field. Adv Interv Cardiol. 2017;13:95-106.

3. Reimers B, Nikas D, Stabile E, Favero L, Saccà S, Cremonesi A, Rubino P. Preliminary experience with optical coherence tomography imaging to evaluate carotid artery stents: safety, feasibility, and techniques. EuroIntervention. 2011;7:98-105.

4. De Donato G, Setacci F, Sirignano P, Galzerano G, Cappelli A, Setacci C. Optical coherence tomography after carotid stenting: rate of stent malapposition, plaque prolapse and fibrous cap rupture according to stent design. Eur J Vasc Endovasc Surg. 2013;45: 579-87.

5. Kotsugi M, Takayama K, Myouchin K, Wada T, Nakagawa I, Nakagawa H, Taoka T, Kurokawa S, Nakase H, Kichikawa K. Carotid artery stenting: investigation of plaque protrusion incidence and prognosis. JACC Cardiovasc Interv. 2017;10:824-31.

6. Bryniarski KL, Tahk SJ, Choi SY, Soeda T, Higuma T, Yamamoto E, Xing L, Dai J, Zanchin T, Lee H, Jang IK. Clinical, angiographic, IVUS and OCT predictors for irregular protrusion after coronary stenting. EuroIntervention. 2017;12:e2204-e2211.

7. Musialek P. Mesh-Covered Stents for Carotid Intervention: Rationale, Device Designs, Imaging, and Data to Date. In: Carotid and Stroke Intervention - State of the Art. TCT, October 14, 2015. Available at http://www.tctmd.com/show.aspx?id=132421 (accessed 20 October 2017)

8. Wissgott C, Schmidt W, Brandt C, Behrens P, Andresen R. Preliminary Clinical Results and Mechanical Behavior of a New Double-Layer Carotid Stent. J Endovasc Ther. 2015;22:634-9.

9. Wissgott C, Schmidt W, Brandt-Wunderlich C, Behrens P, Andresen R. Clinical Results and Mechanical Properties of the Carotid CGUARD Double-Layered Embolic Prevention Stent. J Endovasc Ther. 2017;24:130-137.

10. Schofer J, Musiałek P, Bijuklic K, Kolvenbach R, Trystula M, Siudak Z, Sievert H. A Prospective, Multicenter Study of a Novel Mesh-Covered Carotid Stent: The CGuard CARENET Trial (Carotid Embolic Protection Using MicroNET). JACC Cardiovasc Interv. 2015;8:1229-34.

11. Musialek P, Mazurek A, Trystula M, Borratynska A, LesniakSobelga A, Urbanczyk M, Banys RP, Brzychczy A, Zajdel W, Partyka L, Zmudka K, Podolec P. Novel PARADIGM in carotid revascularisation: Prospective evaluation of All-comer peRcutaneous cArotiD revascularisation in symptomatic and Increased-risk asymptomatic carotid artery stenosis using CGuard ${ }^{\mathrm{TM}}$ MicroNetcovered embolic prevention stent system. EuroIntervention. 2016;12:e658-70.

12. Umemoto $\mathrm{T}$, de Donato $\mathrm{G}$, Pacchioni A, Reimers $\mathrm{B}$, Ferrante G, Isobe M, Setacci C. Optical coherence tomography assessment of new-generation mesh-covered stents after carotid stenting. EuroIntervention. 2017;13:1348-55.

13. Nerla R, Castriota F, Micari A, Sbarzaglia P, Secco GG, Ruffino MA, de Donato G, Setacci C, Cremonesi A. Carotid artery stenting with a new-generation double-mesh stent in three highvolume Italian centres: clinical results of a multidisciplinary approach. EuroIntervention. 2016;12:e677-83.

14. Liu L, Gardecki JA, Nadkarni SA, Toussaint JD, Yagi Y, Bouma BE, Tearney GJ. Imaging the subcellular structure of human coronary atherosclerosis using micro-optical coherence tomography. Nat Med. 2011;17:1010-4.

15. Zapotoczny B, Szafranska K, Owczarczyk K, Kus E, Chlopicki S, Szymonski M. Atomic force microscopy reveals the dynamic morphology of fenestrations in live liver sinusoidal endothelial cells. Sci Rep. 2017;7:7994. 